
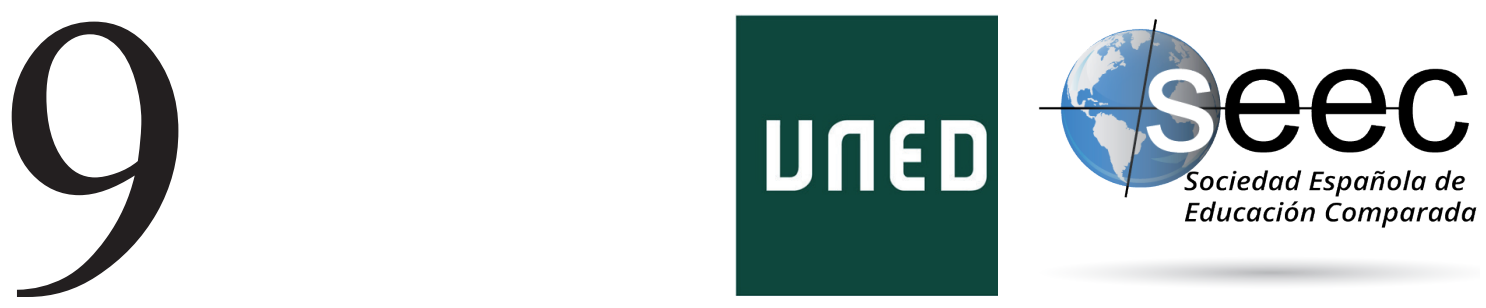

\title{
La Docencia Virtual o e-Learning como Solución a la Enseñanza de la Física y Química de los Futuros Maestros en tiempos de COVID-19
}

\author{
Virtual Teaching or e-Learning as a Solution \\ to the Teaching of Physics and Chemistry of \\ Future Teachers in times of COVID-19
}

\section{Sergio Tirado Olivares*; Ana M. Vázquez ${ }^{* *}$; Rosa M. Toledano***}

DOI: 10.5944/reec.38.2021.28853

\section{Recibido: 8 de noviembre de 2020 Aceptado: 22 de diciembre de 2020}

\footnotetext{
* Sergio Tirado Olivares: Graduado en Maestro en Educación Primaria. Máster en Investigación e Innovación Educativa. Ha sido becario de colaboración en el Departamento de Química-Fisica de la Facultad de Educación de Albacete (UCLM) por el Ministerio de Educación. Datos de contacto: E-mail: Sergio. Tirado@alu.uclm.es.

**Ana M. VÁzquez: Catedrática de Química-Física y profesor de la Facultad de Educación de Albacete de la Universidad de Castilla-La Mancha (UCLM). Su principal campo de investigación se desarrolla en la cromatografía, pero además desarrolla otra línea de investigación relacionada con la enseñanza-aprendizaje de la física y la química. Su labor investigadora ha dado lugar a más de 70 artículos publicados en revistas indexadas de alto impacto y 5 tesis doctorales dirigidas. Es autora de 7 patentes, ha participado y dirigido numerosos proyectos de investigación y ha impartido cursos y conferencias en diversas universidades internacionales. Datos de contacto: E-mail: ana.vazquez@uclm.es. Orcid: http://orcid.org/oooo-0001-5023-4663

\begin{abstract}
***Rosa M. Toledano: PhD, es investigadora y profesora asociada de Química-Física en la Facultad de Educación de Albacete de la Universidad de Castilla-La Mancha (UCLM). Su tesis fue premiada con el Premio Extraordinario de Doctorado otorgado por la UCLM, para el curso académico 2012/13. Su principal campo de investigación se centra en el desarrollo de métodos analíticos. En los últimos años, también desarrolla una línea de investigación centrada en la enseñanza-aprendizaje de las ciencias experimentales. Es coordinadora académica del Grado en Educación Primaria (inglés). Ha realizado etapas de investigación en el Consejo Superior de Investigaciones Científicas (CSIC), en el Laboratorio de Control de Dopaje de Madrid y en la Universidad de Amsterdam (UvA). Datos de contacto: E-mail: rosam.toledano@uclm.es. ORCID: http://orcid.org/oooo-0o03-2376-9341
\end{abstract}




\title{
Resumen
}

Durante el curso académico 2019-20, la tradicional docencia universitaria presencial se vio interrumpida a causa del estado de alarma decretado por el gobierno ante la pandemia SARS-CoV-2. Debido a ello, una nueva docencia no presencial a través de internet (e-Learning) debía ponerse en práctica a contrarreloj con los recursos disponibles. Los docentes de la asignatura de Física y Química impartida en el grado de Maestro en Educación Primaria de la Facultad de Educación de Albacete, adaptamos el temario, los materiales y recursos, la metodología y el sistema de evaluación a la nueva situación. Plataformas como Microsoft Teams, Moodle o Kahoot, pasaron de ser utilizados en momentos puntuales a ser herramientas continuas y fundamentales para trabajar. Con el objetivo de conocer la visión de los estudiantes sobre la metodología y el sistema de evaluación empleado ante esta nueva situación, se elaboró un cuestionario con diferentes bloques de ítems para que el alumnado respondiera a través de una escala Likert de 6 opciones. Las respuestas se evaluaron a través de un análisis estadístico descriptivo y un análisis inferencial para conocer si había diferencias significativas por género mediante el paquete estadístico SPSS vs.24. Los resultados evidencian un elevado grado de satisfacción del alumnado que reconoce y aprecia el esfuerzo extra realizado por los docentes y consideran que las presentaciones confeccionadas, las exposiciones a través de Microsoft Teams, y los foros habilitados para el debate en Moodle, han sido de gran ayuda para el estudio de la materia. Además, no consideran que esta situación haya influido en su logro académico. Del estudio realizado por género se extrae una gran equidad en las respuestas. No obstante, el alumnado femenino se siente más incómodo con la docencia a través de las nuevas tecnologías y que su uso, tanto durante las sesiones de clase como durante las pruebas evaluativas, les genera mayor estrés que al alumnado masculino.

Palabras clave: Docencia virtual; e-learning; Pandemia; Física y Química; Futuros maestros.

\begin{abstract}
During the 2019-20 academic year, the traditional face-to-face university teaching was interrupted due to the state of alarm decreed by the government in the face of the SARS-CoV-2 pandemic. Because of that, a new non-face-to-face teaching through internet (e-Learning) had to be put into practice with the available resources quickly. The teachers of the Physics and Chemistry course taught in the Primary Education Degree of the Faculty of Education in Albacete, had to adapt the contents, materials and resources, but also the methodology and the evaluation system to the new situation. Platforms such as Microsoft Teams, Moodle or Kahoot, went from being used at specific moments to being continuous and fundamental teaching tools. To know the students' vision about the methodology and the evaluation system used in this new situation, a questionnaire was elaborated with different blocks of items to be responded through a Likert scale of 6 options by the students. The responses were evaluated through a descriptive statistical analysis and an inferential statistical analysis was carried out to know if there were significant differences by gender using the statistical package SPSS vs.24. The results show a high degree of satisfaction among the students who recognize and appreciate the extra effort made by the teachers. They also consider that the presentations made, the lessons through Microsoft Teams, and the debate forums enabled in Moodle, have been useful for the study of the course. Furthermore, students do not consider that this situation has influenced their academic achievement. The study carried out by gender showed a great fairness. However, female students feel more uncomfortable with the teaching through new technologies. Moreover, their use both, during the lessons and during evaluative tests, causes higher stress levels in female than in male students.
\end{abstract}

Keywords: Virtual teaching; e-learning; Pandemic; Physics and Chemistry; Future teachers. 


\section{Introducción}

\subsection{Docencia en la Universidad de Castilla-La Mancha durante la pandemia}

Durante el curso académico 2019-20, más concretamente a mediados del segundo cuatrimestre, la docencia universitaria presencial a la que estábamos acostumbrados tanto estudiantes como profesores, se vio drástica y bruscamente interrumpida a causa del estado de alarma decretado por el gobierno de España el día 14 de marzo (RD 463/2020, 2020), ante la situación de pandemia (COVID-19) producida por el SARS-CoV-2. El rector de la Universidad de Castilla-La Mancha (UCLM), Miguel Ángel Collado, en una resolución del 11 de marzo de 2020 suspendió toda actividad presencial en el ámbito de la UCLM durante 15 días (Collado Yurrita, 2020). La vicerrectora de docencia emitió una resolución para adaptar la universidad a la suspensión temporal de la actividad educativa presencial en la que se indicaba que todos los cambios de programación de las asignaturas, sistemas de evaluación, régimen de tutorías deberían comunicarse a los estudiantes a través de Campus Virtual y se recomendaba utilizar los medios disponibles en la Universidad para la docencia a distancia: Microsoft Teams para clases y tutorías de manera sincrónica y el empleo de los recursos disponibles en Campus Virtual (LópezSolera, 2020). En un principio, todos pensamos que pronto volveríamos a la situación de normalidad, sin embargo, fuimos viendo como prorroga tras prorroga el estado de alarma continuaba en nuestro país. El 2 de abril, la UCLM suspendió definitivamente las clases presenciales en este curso y adoptó el régimen de docencia no presencial para concluir las clases y los exámenes en el curso académico 2019/20. El Vicerrectorado de Docencia desarrolló diversas alternativas con el fin de establecer un marco flexible que permitiese a los profesores adoptar las medidas que considerasen más adecuadas para el desarrollo de las actividades de aprendizaje y evaluación, teniendo en cuenta las características específicas de sus asignaturas y las directrices dadas por el Ministerio de Universidades (Ministerio de Universidades, 2020). Así mismo, con el objeto de asegurar que el estudiantado pudiera finalizar sus estudios, con las adaptaciones que fuesen necesarias, se emitieron directrices académicas de adaptación de la evaluación presencial a la no presencial (UCLM, 2020), en la que se consideraba la realización tanto de pruebas asincrónicas (realizadas no simultáneamente por todos los estudiantes y sin la presencia del profesor) como sincrónicas (en las que el conjunto de estudiantes se conecta simultáneamente durante un mismo espacio de tiempo y con la supervisión en línea del profesor).

Los profesores y estudiantes tuvimos que adaptarnos a la nueva situación. El cambio abrupto de una docencia presencial planificada, a una docencia en línea, no puede compararse a un planteamiento en cuanto métodos y recursos ya preestablecida en línea (Hodges et al., 2020). En una Universidad de vocación eminentemente presencial, como la UCLM, en la que el contacto personal entre todos los que integramos la comunidad educativa es de gran importancia, el impacto de la situación creada por el COVID-19 fue muy superior al que pudo producirse en otras universidades en las que la docencia online es parte de su esencia como la UNED (Universidad Nacional Educación a Distancia) o la UOC (Universitat Oberta de Catalunya). Los profesores estábamos acostumbrados a impartir las clases presenciales en el aula, utilizando las presentaciones de PowerPoint, elaboradas para ir explicando los contenidos al tiempo que se proyectan y no para el aprendizaje autónomo de los alumnos, al uso de la pizarra, del laboratorio y sobre todo a 
la comunicación tanto verbal como no verbal (lenguaje corporal) con nuestros alumnos. La forma y manera de interactuar en el aula era un aspecto fundamental en el proceso de enseñanza-aprendizaje. La comunicación no verbal nos permitía transmitir actitudes hacia los contenidos, atraer la atención de nuestros alumnos, conocer sus estados emocionales (cansancio, interés-desinterés, aburrimiento, etc.) (Álvarez, 2012). En la docencia no presencial, el lenguaje no verbal pierde todo su valor. En definitiva, estábamos acostumbrados a una forma de impartir nuestras clases que tuvimos que modificar prácticamente de la noche a la mañana.

Si bien es cierto que tanto los estudiantes como el profesorado estábamos familiarizado con el uso de plataformas de e-learning, como Moodle (González, 2006; Pruneda et al., 2019) base del Campus Virtual de la UCLM, también es verdad que la utilizábamos más como una herramienta de apoyo a la enseñanza presencial que nos permitía poner a disposición de los alumnos materiales y recursos y solicitar tareas, y no realmente como una plataforma de enseñanza no presencial. Los estudiantes usaban esta plataforma principalmente para consultar apuntes, subir trabajos y para informarse sobre notas de exámenes (Sánchez Santamaría et al., 2012).

La UCLM disponía de los recursos tecnológicos necesarios para poder afrontar la nueva situación y se organizaron cursos y seminarios online con el objetivo de formar al profesorado. En un espacio de tiempo muy corto, los profesores tuvimos que cambiar nuestra metodología docente, tuvimos que formarnos a través de tutoriales y cursos online en el uso de nuevas tecnologías de la información y la comunicación que nos permitieran crear un entorno virtual de aprendizaje para nuestros alumnos (García-Planas y Taberna Torres, 2020). En definitiva, la situación llevó, de manera obligatoria, al profesorado a un aprendizaje online o e-learning y para el que nos tuvimos que preparar a contrarreloj. El contexto social propició pasar de la tecnología como herramienta de apoyo a ser la herramienta fundamental para una educación online o e-learning de manera generalizada y continua (Torrecillas, 2020). De acuerdo con recientes estudios parece ser que, en primera instancia, y teniendo en cuenta la rapidez con la que se han tenido que poner en práctica, las adaptaciones realizados en los planes docentes pueden considerarse aceptables (García-Peñalvo, et al, 2020).A pesar de las controversias e incertidumbre que la enseñanza online despierta en gran parte de la comunidad educativa, su utilización presenta algunas ventajas educativas. Se ha demostrado que su uso permite la ruptura de las barreras espaciotemporales; flexibilizando la educación y permitiendo una mayor personalización y contextualización de la educación acorde con la actual sociedad de la comunicación. De este modo, el e-learning permite compatibilizar mejor la vida cotidiana con el proceso de enseñanza-aprendizaje (Hurt e Ivergard, 2008; Peñalvo, 2005).

Diversos modelos pedagógicos, basados en el uso de internet, han sido analizados y puestos en práctica. Autores como Alshammari (2020) evidencian que este nuevo planteamiento pedagógico habilita entornos de aprendizajes adaptativos y autorregulados, pues propician la aparición de un nuevo agente educador computacional con retroalimentación continua y rápida del logro académico alcanzado. En esta línea, PensalbeRodríguez et al. (2020) han comprobado que plataformas virtuales, confeccionadas a modo de red social, permiten una mejora significativa de la actitud del alumnado y facilitan el uso de espacios de debate, sistemas de mensajería directa alumno-profesor, y las herramientas necesarias para el planteamiento de actividades y su envío, una vez realizadas, telemáticamente. Es indudable que la enseñanza virtual fomenta más 
la autonomía, la visión crítica y la capacidad de autoaprendizaje de los estudiantes y posibilita el «metaaprendizaje» generando una reflexión cognitiva de los estudiantes (Herrera y Pérez, 2002).

Sin embargo, la controversia de su uso es evidente. A pesar de que su uso se haga con previsión, autores como Sánchez (2018) evidencian que su uso (sobre todo en niveles como Educación Primaria), pese a fomentar el trabajo cooperativo y la motivación, requieren un largo trabajo formativo del profesorado, adaptación de los materiales y, sobre todo, es imprescindible que los estudiantes dispongan de los dispositivos (móviles, ordenadores, tablet, etc.) y conectividad necesaria. También los estudiantes y el profesorado deben disponer, en sus viviendas, de un espacio en el cuál puedan conectarse sin ser interrumpidos por la vida familiar, cosa que muy frecuentemente no se da ya que nuestras viviendas no están diseñadas para ello. Este es el motivo por el cual tales adaptaciones pedagógicas deben hacerse ligadas a guías creadas a contrarreloj para tal fin (García-Peñalvo, et al, 2020); al igual que siguiendo las recomendaciones institucionales de flexibilidad en los planteamientos evaluativos para garantizar la inclusión de todo el alumnado (UNESCO, 2020). Es este el único modo con el que podemos asumir ciertas garantías de éxito.

En nuestro caso concreto, el cambio de una enseñanza presencial a una enseñanza virtual supuso también la adaptación de los contenidos, actividades, temporalización, sistema y criterios de evaluación de cada una de las asignaturas al tiempo que se intentó garantizar que los alumnos alcanzarán los objetivos y las competencias que se incluían en las guías docente de las diferentes asignaturas.

Los profesores del departamento de Química-Física de la Facultad de Educación de Albacete de la UCLM mantuvimos continuas reuniones a través de Microsoft Teams, discutimos y acordamos los cambios que íbamos a realizar en los contenidos, elaboramos nuevos materiales que facilitaran el aprendizaje autónomo de los alumnos, sustituimos las actividades presenciales por otras que pudieran ser realizadas a través de entornos virtuales y elaboramos nuevos criterios de evaluación que se pusieron inmediatamente en conocimiento de los alumnos a través de la plataforma Moodle. Todas estas actividades supusieron una sobrecarga de trabajo para el profesorado, al igual que le ocurrió al profesorado de otros centros docentes y universidades (Silas Casillas y Vázquez Rodríguez, 2020). Silas y Vázquez (2020) han realizado un estudio sobre la vivencia del profesorado universitario del proceso de transición forzada de la docencia presencial a la docencia virtual y concluyeron que los profesores llevaron a cabo este proceso basándose en sus intuiciones y experiencias docentes, acompañados en la mayoría de los casos de algún tipo de formación institucional y/o personal y que está nueva situación generó mucha frustración y estrés en los docentes y una considerable sobrecarga de trabajo.

\subsection{La asignatura de El medio natural I: física, química y su didáctica}

La asignatura «El medio natural I: física, química y su didáctica» es una asignatura obligatoria, de 6 créditos que se imparte en el segundo cuatrimestre del tercer curso del grado de Maestro en Educación Primaria. El plan de estudios del grado consta de un bloque de formación básica y otro didáctico disciplinar, además del prácticum y el TFG. La enseñanza/aprendizaje de las Ciencias Experimentales está incluida en el bloque didáctico disciplinar con total de 15 créditos, para la impartición de todas las disciplinas científicas y su didáctica. «El medio natural I: física, química y su didáctica» es la única asignatura del plan de estudios dedicada a dos de las más importantes ciencias de la naturaleza (física y química) fundamentales para entender y avanzar en el mundo 
científico-tecnológico en el que los futuros maestros deberán desenvolverse y para poder abordar, en su futura vida profesional, los contenidos que se desarrollan en el curriculum de Educación Primaria (BOE, 2014). Con esta perspectiva, el programa de la asignatura fue elaborado con el objetivo de desarrollar los contenidos de física y química necesarios en el desarrollo de la vida profesional del «futuro maestro», y teniendo en cuenta que el programa debía ser abordable en un curso académico, considerando además, el muy desigual conocimiento de estas disciplinas por los estudiantes, ya que nos encontramos con estudiantes que han cursado el bachillerato científico-tecnológico junto otros de muy diversas procedencias.

El programa de la asignatura está dividido en 3 bloques, el primero de Introducción a la física y la química, y los y los otros dos bloques están dedicado cada uno de ellos a una de estas disciplinas. Los temas incluidos en cada uno de los bloques están descritos en la Tabla 1.

Tabla 1.

Descripción de los bloques y contenidos didácticos de la asignatura «El medio natural I: física y química y su didáctica»

\begin{tabular}{|c|c|}
\hline Bloque & Tema \\
\hline $\begin{array}{l}\text { Bloque I. } \\
\text { Introducción } \\
\text { a la Física y la } \\
\text { Química }\end{array}$ & $\begin{array}{l}\text { Tema 1: La física y la química ciencias experimentales } \\
\text { Campo de estudio de la física y de la química } \\
\text { El surgimiento de la física como ciencia: de la Astrología a la Astronomía } \\
\text { El surgimiento de la química como ciencia: de la Alquimia a la química } \\
\text { moderna } \\
\text { Tema 2: La medida de los cuerpos y sus unidades } \\
\text { Magnitudes físicas. Medida de una magnitud física. Sistemas de Unidades } \\
\text { Cambio de unidades. Factor de conversión. Ecuación de dimensiones }\end{array}$ \\
\hline \multirow[t]{4}{*}{$\begin{array}{l}\text { Bloque II. } \\
\text { Química }\end{array}$} & $\begin{array}{l}\text { Tema 3. ¿Qué es la materia? } \\
\text { Sistemas materiales. Propiedades de la materia. Sistemas heterogéneos y } \\
\text { sistemas homogéneos. Sustancias puras. Mezclas. Elementos. Compuestos } \\
\text { Disoluciones. Sistemas dispersos }\end{array}$ \\
\hline & $\begin{array}{l}\text { Tema 4. Separación de sustancias } \\
\text { Separación de los componentes de una mezcla } \\
\text { Filtración. Decantación. Centrifugación. Destilación. Cristalización. } \\
\text { Cromatografía }\end{array}$ \\
\hline & $\begin{array}{l}\text { Tema 5. Estados de agregación de la materia } \\
\text { Estados de agregación de la materia: gas, líquido y sólido. Cambios de } \\
\text { estado }\end{array}$ \\
\hline & $\begin{array}{l}\text { Tema 6. Cambios químicos de nuestro entorno } \\
\text { Las reacciones químicas. Ecuaciones químicas. Reacciones químicas y } \\
\text { energía. } \\
\text { Reacciones de oxidación, combustión y fermentación. Reacciones químicas y } \\
\text { medio ambiente }\end{array}$ \\
\hline \multirow[t]{3}{*}{$\begin{array}{l}\text { Bloque III. } \\
\text { Física }\end{array}$} & $\begin{array}{l}\text { Tema 7. Electricidad y Magnetismo } \\
\text { Cargas eléctricas. Aislantes y conductores. Corriente eléctrica. Circuitos } \\
\text { eléctricos. Magnetismo. Relación entre electricidad y magnetismo. }\end{array}$ \\
\hline & $\begin{array}{l}\text { Tema 8. Las Fuerzas y sus efectos } \\
\text { Concepto de Fuerza. Leyes de Newton. Principio de Gravitación Universal. } \\
\text { Caída libre de los cuerpos. Flotabilidad en un medio líquido }\end{array}$ \\
\hline & $\begin{array}{l}\text { Tema 9. El equilibrio de los cuerpos y su aplicación tecnológica } \\
\text { Equilibrio de un cuerpo. Equilibrio de traslación y de rotación. Máquinas } \\
\text { simples: palanca, polea, plano inclinado y torno }\end{array}$ \\
\hline
\end{tabular}


En el momento de decretarse el estado de alarma, se había terminado de impartir el tema 4: separación de sustancias, que es un tema eminentemente práctico y que incluye la realización de prácticas de laboratorio e íbamos a comenzar el tema 5: estados de agregación de la materia.

El objetivo del presente trabajo es mostrar cómo nos enfrentamos los profesores del departamento de Química-Física de la Facultad de Educación de Albacete de la UCLM a la nueva situación para la docencia de la asignatura «El medio natural I: física, química y su didáctica», de tercer curso del grado de Maestro en Educación Primaria y cómo percibieron los alumnos los cambios introducidos. Este objetivo lo podemos desglosar en los siguientes objetivos específicos:

- Adaptar los contenidos de la asignatura de Física y Química para la formación de futuros docentes de Educación Primaria a la nueva situación de excepcionalidad de manera rápida y eficaz para una formación correcta de todos los estudiantes.

- Planificar y desarrollar un planteamiento pedagógico a través de metodologías innovadoras y en línea para continuar con la docencia de manera eficaz y cumpliendo los objetivos establecidos

- Analizar la visión del alumnado ante la metodología y sistema de evaluación impartidos, actitud y esfuerzo del profesorado y su propia vivencia.

\section{Materiales y métodos}

\subsection{Adaptación de la docencia}

Para adaptar la docencia de la asignatura nos planteamos utilizar el modelo docente de clase invertida (Flipped Classroom), combinado con e-learning, de manera que se fomentará la autonomía del alumno y su capacidad de autoaprendizaje. Se modificaron los documentos y las presentaciones que se iban a poner a disposición de los alumnos. Se ampliaron las explicaciones incluidas en dichos documentos y se incluyeron enlaces a videos explicativos a través del sitio web YouTube. Estos materiales se fueron subiendo gradualmente y de forma escalonada en el tiempo a la plataforma Moodle, con el objetivo de no saturar al alumno y de dirigir su aprendizaje en el tiempo. Se planificaron actividades asincrónicas que debían ser realizadas por los estudiantes (resolución de problemas, actividades experimentales sencillas que podían ser realizadas en casa, búsqueda de información, uso de simuladores a través de la plataforma TIC Phet Colorado, actividades de autoevaluación creadas por los profesores con la plataforma Kahoot!, etc..) con las que se crearon tareas en la plataforma Moodle que debían ser entregadas por los estudiantes en unas fechas prefijadas. Se abrieron foros en los que se planteaban debates y dudas que iban surgiendo. Finalmente se mantuvieron los horarios de las clases utilizando estas horas para encuentros sincrónicos con los estudiantes a través de Teams, que permitieran la resolución de dudas por parte del profesor y la participación de los estudiantes.

Creíamos que era muy importante mantener el contacto con nuestros alumnos (aunque fuese de forma virtual) de manera que sintieran que no estaban solos en su proceso de aprendizaje, que contaban con el apoyo del profesorado y que podían mantener la interacción entre iguales. Así mismo, el profesorado estuvimos en todo momento a 
disposición de nuestros alumnos para resolver las dudas que iban surgiendo a través de tutorías personalizadas en Teams o por correo electrónico. Los alumnos fueron informados de todas las decisiones que íbamos tomando los profesores del departamento a través de notas que se colgaban en Moodle. Terminado el periodo de formación, los alumnos realizaron una prueba final a través de Moodle de forma sincrónica. Dicha prueba se preparó gracias a la herramienta cuestionarios de Moodle. Se crearon bancos de preguntas relacionados con los distintos temas de la asignatura. Los tipos de preguntas variaron desde la opción múltiple, la respuesta corta, o la resolución de problemas. Para facilitar su respuesta y minimizar el estrés asociado a la posibilidad de un fallo en la red, se permitió que pudiesen hacer las preguntas cortas y problemas en papel y pudiesen adjuntar la solución como un archivo adjunto a la pregunta. Se crearon reuniones por Teams durante el examen de tal forma que los alumnos pudiesen comunicar instantáneamente sus dudas o incidencias.

Además, los profesores del departamento tuvimos que adaptar el plan de trabajo. En primer lugar, se llevó a cabo una modificación del temario pues considerábamos que en la nueva situación era prácticamente imposible abarcar los contenidos incluidos en el programa. Se redujeron los contenidos del tema 5, tema 6 (bloque II: química) y del tema 7 (Bloque III: física). El tema 8 se eliminó del temario y los contenidos de este tema imprescindibles se incluyeron en el tema 9.

Por otra parte, se modificaron los criterios de evaluación y se adaptaron a la nueva situación. Se aumentó significativamente el peso de las actividades realizadas con detrimento del peso de la calificación del examen, de acuerdo con las directrices dadas por el vicerrectorado de docencia de la UCLM. De tal forma que, aunque se mantuvo la condición de calificación mínima en la prueba final (igual o superior a un 4 sobre 10), se redujo el peso de la prueba final de un 70 a un 50 \% de la calificación final, quedando el sistema de evaluación como se muestra en la Tabla 2.

Tabla 2.

Comparación del sistema de evaluación de la asignatura antes y durante la situación de pandemia

\begin{tabular}{lllll}
\hline & \multicolumn{2}{l}{ Antes pandemia } & \multicolumn{2}{l}{ Durante la pandemia } \\
\hline Tipo Trabajo & \% Calificación & Modalidad & \% Calificación & Modalidad \\
\hline Trabajo Tema 1 & 10 & Grupo & 10 & Grupo \\
Cuaderno Laboratorio & 10 & Grupo & 10 & Grupo \\
Actividades Tema 6 & & No se realizaba & 5 & Individual \\
$\begin{array}{l}\text { Cuestión reacción } \\
\text { la vida cotidiana }\end{array}$ & No se realizaba & 5 & Individual \\
Control (Tema 7) & & No se realizaba & 10 & Individual \\
Control (Tema 9) & & No se realizaba & 10 & Individual \\
PRUEBA FINAL & 70 & Individual & 50 & Individual \\
\hline
\end{tabular}

\subsection{Participantes}

El número total de estudiantes que cursaron la asignatura en curso 19/20 fue 170, distribuidos en tres grupos de clase. En dos de estos grupos la docencia se impartió en castellano (grupo A y C) y en el tercero, grupo bilingüe, la docencia se impartió en inglés (grupo B). En la tabla 3, puede verse el número de alumnos por grupo y su distribución por género. 
Tabla 3.

Estudiantes participantes en la asignatura, su distribución por grupos de clase y por género

\begin{tabular}{cccc}
\hline Grupo de Clase & Chicos & Chicas & Total de alumnos \\
\hline A & 26 & 48 & 74 \\
B & 9 & 12 & 21 \\
C & 34 & 41 & 75 \\
\hline
\end{tabular}

Una vez finalizada la docencia y evaluación de los estudiantes se procedió a realizar un análisis cuantitativo con medida post-test para conocer cómo habían afrontado la situación generada por la pandemia COVID-19, su opinión sobre la actuación del profesorado de la asignatura y sobre cómo se había adaptado la misma a la docencia online. Para conocer esta visión de los estudiantes, se confeccionó un cuestionario digital. El cuestionario se elaboró a través de la herramienta Microsoft Forms y se generó un enlace que se les envió a los estudiantes a través del campus virtual (Moodle) de la asignatura. Se optó por la herramienta Microsoft Forms porque permite una fácil distribución del cuestionario (correo electrónico, generación de código QR, o link), se pueden completar en un ordenador o través de los dispositivos móviles (tablet o teléfonos móviles). Los estudiantes fueron informados del carácter anónimo y voluntario del cuestionario, para el cuál no se especificó un límite de tiempo de respuesta. De los 170 estudiantes de la asignatura, 71 completaron el cuestionario (Tabla 4).

Tabla 4.

Estudiantes que completaron el cuestionario, su distribución por grupos de clase y por género

\begin{tabular}{cccc}
\hline Grupo de Clase & Chicos & Chicas & Total de alumnos \\
\hline A & 13 & 30 & 43 \\
B & 3 & 5 & 8 \\
C & 5 & 15 & 20 \\
\hline
\end{tabular}

Cabe destacar que la diferencia entre el número de respuestas de chicos y chicas que respondieron el cuestionario es notable. Esto es debido a la tradición existente en el grado de educación, pues la elección de estudios de Educación es realizada mayoritariamente por alumnado femenino (Navarro y Casero, 2012), pero también puede deberse a que al ser voluntario la realización del cuestionario, ya que mayoritariamente las chicas presentan una mayor predisposición a la realización de actividades no obligatorias.

\subsection{Instrumento}

El cuestionario ad hoc, instrumento de recogida de datos, puede verse en la Tabla 5 . El cuestionario se dividió en diversos bloques, que buscaban conocer, por un lado, la opinión de los alumnos sobre la planificación y desarrollo de la docencia adaptada: Planificación y desarrollo de la docencia (PD) (Bloque 1), Recursos utilizados (RU) (Bloque 2), Actividades (ACT) (Bloque 3) y Evaluación (EV) (Bloque 4). Además, se incluyó un bloque de preguntas para conocer su opinión sobre la Actitud del profesorado (AP) (Bloque 5) y una autoevaluación personal, Cómo me he sentido durante este proceso (VE) (Bloque 6). Inicialmente se incluyó un bloque específico (Bloque 7) de opinión sobre la docencia en inglés, que finalmente no se tuvo en cuenta en el estudio ya que el 
número de participantes del grupo B fue muy pequeño. El inicio del cuestionario incluyó tres cuestiones clasificatorias sobre grupo de clase, género y edad, y al final del cuestionario, tres ítems de valoración global.

Tabla 5 .

Indicaciones e ítem incluidos en el cuestionario digital

Se recuerda que toda la información obtenida de esta encuesta será totalmente anónima y se tratará exclusivamente con fines estadísticos.

\begin{tabular}{|c|c|c|c|c|c|}
\hline GRUPO DE CLASE & Grupo A & & Grupo B & & Grupo C \\
\hline SEXO BIOLÓGICO & Hombre & \multicolumn{4}{|c|}{ Mujer } \\
\hline EDAD & Menor de 20 & 21 a 30 & 21 a 40 & 41 a 50 & Mayor de 61 \\
\hline \multicolumn{6}{|c|}{$\begin{array}{l}\text { VALORA SEGÚN LA SIGUIENTE ESCALA 1-5, TU OPINIÓN RESPECTO DE LA AFIR- } \\
\text { MACIÓN CONTENIDA EN CADA ÍTEM. SI NO TIENES UNA OPINIÓN FORMADA, MARCA } \\
\text { LA CASILLA O; NS (No sabe/No contesta). }\end{array}$} \\
\hline \multicolumn{6}{|c|}{$\begin{array}{l}\text { o: No sabe/No contesta (NS); 1: muy en desacuerdo; 2: en desacuerdo; 3: indiferente; 4: de } \\
\text { acuerdo; 5: muy de acuerdo }\end{array}$} \\
\hline
\end{tabular}

\section{Bloque 1. Planificación y desarrollo de la docencia}

PD1. La adaptación del temario a la nueva situación de la docencia ha sido adecuada

PD2. La división de los temas impartidos online en diversas partes me ha permitido seguir mejor los contenidos

PD3. La reducción del temario ha permitido impartirlo en el tiempo disponible

PD4. La docencia online de esta asignatura ha supuesto una sobrecarga de trabajo para los estudiantes

\section{Bloque 2. Recursos utilizados}

RU1. Me ha sido más difícil comprender los conceptos en las clases online (a través de Teams) que en las clases presenciales

RU2. He tenido problemas para conectarme a las clases con Teams

RU3. El material puesto a disposición en la plataforma Moodle en el periodo de docencia online me ha facilitado el seguimiento de la asignatura

RU4. La comunicación del profesorado con los estudiantes a través de Moodle y Teams ha sido adecuada

RU5. La participación de los alumnos en las clases online ha sido más difícil que en las clases presenciales

RU6. Estoy satisfecho con el funcionamiento de la plataforma Moodle durante la docencia no presencial

RU7. Los videos incluidos en las presentaciones me han ayudado a comprender los conceptos de la asignatura

\section{Bloque 3. Actividades}

ACT1. Las actividades planificadas para la docencia online (clases a través de Teams, cuestionarios, problemas, ...) me han parecido suficientes y adecuadas

ACT2. El empleo de Kahoot al finalizar cada tema me ha permitido realizar una autoevaluación de mi progreso

ACT3. Los problemas han sido más difíciles de comprender que en las clases presenciales

ACT4. Los PowerPoint utilizados me han sido de utilidad para comprender los conceptos

ACT5. El tiempo asignado para la realización de las actividades ha sido adecuado

ACT6. Los foros habilitados me han ayudado a resolver las dudas 


\section{Bloque 4. Evaluación}

EV1. La metodología de evaluación (50 \% evaluación continua y 50 \% evaluación examen) me parece adecuada

EV2. Los criterios de evaluación modificados han sido claramente comunicados y explicados por el profesorado

EV3. La realización del examen online ha sido complicado

EV4. La realización del examen online ha supuesto un estrés adicional

\section{Bloque 5. Actitud del profesorado}

AP1. Considero que el profesorado ha hecho un esfuerzo por reforzar el material puesto a disposición de los alumnos para facilitar el seguimiento de la asignatura (PowerPoint, problemas, actividades....)

AP2. La atención del profesorado mediante el correo electrónico y tutorías personalizadas ha sido adecuada

AP3. El profesor se ha preocupado por saber si se comprendían los conceptos explicados en las clases online

AP4. El profesor ha atendido la demanda de los alumnos siempre que ha sido posible

\section{Bloque 6. Cómo me he sentido durante este proceso (Valoración como estudi-} ante)

VE1. La nueva metodología me ha generado incertidumbre ante cómo estudiar la asignatura

VE2. Considero que mis resultados se verán mejorados debido a la situación actual

VE3. He tenido la infraestructura necesaria para acceder a los recursos y materiales necesarios para seguir la asignatura

VE4. Considero que en un futuro, y con mayor previsión, esta metodología se adecuará mejor a mis necesidades educativas que la metodología seguida en las clases presenciales

\section{VALORACIÓN GLOBAL}

Indica usando una escala del 1 al 10 tu grado de valoración global, siendo el 1 muy insatisfecho y el 10 muy satisfecho.

Considerando todo lo anterior, y a pesar de las dificultades debidas a la situación excepcional del COVID-19:

VG1. La docencia online ha sido planificada correctamente y se ha podido alcanzar los objetivos y competencias de la asignatura

VG2. Estoy satisfecho o satisfecha con el trabajo realizado por el profesorado

VG3. La docencia online no ha hecho más difícil el estudio de la asignatura

En los seis bloques descritos se incluyeron distintos ítems que los estudiantes debían evaluar a través de una escala Likert de o a 5, codificada como 1: muy en desacuerdo; 2: en desacuerdo; 3: indiferente; 4: de acuerdo; 5: muy de acuerdo; o: No sabe/No contesta (NS). Sin embargo, para el bloque de valoración global quisimos conocer el grado de satisfacción por lo que se optó por una escala del 1 al 10, siendo el 1 muy insatisfecho y el 10 muy satisfecho. Además, con el objetivo de evitar el fenómeno de aquiescencia o respuesta automatizada por parte del alumno, ciertos ítems pertenecientes a los diferentes bloques descritos fueron redactados en negativo (PD4; RU2; ACT3; EV3; EV4; VE1; VE2).

Otra de las ventajas de la herramienta Microsoft Forms es que permite exportar a un documento Excel las respuestas recibidas. Sin embargo, el tratamiento estadístico de los datos de este estudio se realizó a través del paquete estadístico SPSS vs. 24. Se llevó a cabo el análisis descriptivo (porcentajes de respuesta en cada opción de los ítems y medias 
aritmética por género). Para determinar si existían diferencias significativas por género, se asumió la distribución normal al ser el tamaño de las muestras mayor a 30, se utilizó el test de Levene para comprobar la homogeneidad de varianzas y se procedió a realizar el test t-student para muestras independientes con un nivel de significación de 0.05.

Para llevar a cabo el análisis de los resultados los bloques de preguntas se agruparon en tres grandes apartados:

1. Visión del alumnado sobre la metodología implementada: incluye los ítems del Bloque 1. Planificación y desarrollo de la docencia y del bloque 2: Recursos utilizados.

2. Visión del alumnado sobre las actividades realizadas y proceso evaluativo establecido: incluye los ítems del Bloque 3. Actividades y del Bloque 4. Evaluación.

3. Percepción del alumnado de la actitud del profesorado y afinidad con el proyecto: incluye las preguntas del Bloque 5. Actitud del profesorado y del Bloque 6. Cómo me he sentido durante este proceso (Valoración como estudiante).

\section{Resultados y discusión}

El análisis de los resultados del cuestionario se presenta de acuerdo con los tres grandes apartados indicados en materiales y método, constituidos cada uno de ellos por dos bloques de preguntas. Al final de cada uno de los apartados se incluye una de las tres preguntas generales expuestas al final del test, en el bloque Valoración global, de tal forma que la pregunta VG1 corresponde al bloque 1; la VG3 al bloque 2 y la VG2 al bloque 3. De este modo, a través de la media de cada una de estas preguntas, se detecta de forma global la satisfacción o no del alumnado con respecto al punto analizado en cada uno de los tres apartados.

\subsection{Visión del alumnado sobre la metodología implementada}

La tabla 6 muestra los resultados obtenidos respecto a la gestión realizada del tiempo, planificación y desarrollo de las sesiones (preguntas PD 1 a 4) y recursos utilizados (preguntas RU 1 a 7). Más del $75 \%$ de los estudiantes se mostraron de acuerdo o muy de acuerdo con la adaptación del temario a la nueva situación (PD1), un 84,6 \% considera que la división de los temas en partes más pequeñas les ha facilitado su estudio (PD2) y hasta el $90 \%$ cree que la reducción del temario ha permitido que se haya impartido en el tiempo disponible (PD3). Además, más del $60 \%$ de los estudiantes no considera que la docencia online haya supuesto una sobrecarga de trabajo para ellos (PD4). Estos resultados indican la visible satisfacción del alumnado respecto a la planificación de la docencia por parte del profesorado. 
Tabla 6.

Análisis descriptivo de las respuestas obtenidas en cada una de las preguntas de los bloques 1: Planteamiento y Desarrollo (PD) y bloque 2: Recursos Utilizados (RU). No sabe/No contesta (NS/NC), muy en desacuerdo (MD), en desacuerdo (D), indiferente (I) de acuerdo (A), muy de acuerdo (MA). El análisis por género ha sido sometido a un análisis inferencial a través de la prueba T.

\begin{tabular}{|c|c|c|c|c|c|c|c|c|c|c|}
\hline \multirow[t]{2}{*}{ Ítem } & \multirow[t]{2}{*}{$\begin{array}{l}\text { NS/NC } \\
(\%)\end{array}$} & \multirow[t]{2}{*}{$\begin{array}{l}\text { MD } \\
(\%)\end{array}$} & \multirow[t]{2}{*}{$\mathrm{D}(\%)$} & \multirow[t]{2}{*}{ I (\%) } & \multirow[t]{2}{*}{ A (\%) } & \multirow[t]{2}{*}{$\begin{array}{l}\text { MA } \\
(\%)\end{array}$} & \multicolumn{2}{|c|}{ Género (X) } & \multirow{2}{*}{$\begin{array}{l}\text { T-test } \\
\text { Valor } \\
\end{array}$} & \multirow{2}{*}{$\begin{array}{l}\text { Sig } \\
\mathrm{P}\end{array}$} \\
\hline & & & & & & & 0 & 우 & & \\
\hline PD1 & & 1,4 & 7,0 & 16,9 & 32,4 & 42,3 & 4,48 & 3,9 & $\mathbf{2 , 2 7}$ & ,026 \\
\hline PD2 & 1,4 & 2,8 & & 11,3 & 32,4 & 52,1 & 4,24 & 4,28 &,- 156 & ,877 \\
\hline PD3 & & 1,4 & 2,8 & 5,6 & 39,4 & 50,7 & 4,57 & 4,26 & 1,455 & ,15 \\
\hline PD4 & 5,6 & 31 & 31 & 19,7 & 5,6 & 7,0 & 1,76 & 2,24 & $-1,463$ & ,148 \\
\hline $\mathrm{PD}(\mathrm{X})$ & & & & & & & 4,31 & 3,96 & 1,829 & ,072 \\
\hline RU1 & 2,8 & 23,9 & 19,7 & 12,7 & 19,7 & 21,1 & 2,33 & 3,08 & $-1,861$ & ,067 \\
\hline RU2 & 9,9 & 43,7 & 11,3 & 12,7 & 16,9 & 5,6 & 1,95 & 2,02 &,- 175 &, 861 \\
\hline $\mathrm{RU}_{3}$ & & & 4,2 & 14,1 & 26,8 & 54,9 & 4,48 & 4,26 & ,95 &, 345 \\
\hline RU4 & & & & 2,8 & 23,9 & 73,2 & 4,71 & 4,7 & ,105 & ,916 \\
\hline RU5 & 1,4 & 12,7 & 12,7 & 19,7 & 26,8 & 26,8 & 2,95 & 3,56 & $-1,681$ & ,097 \\
\hline RU6 & 1,4 & 8,5 & 9,9 & 25,4 & 29,6 & 25,4 & 3,67 & 3,42 & ,735 & ,465 \\
\hline RU7 & 1,4 & 4,2 & 1,4 & 11,3 & 35,2 & 46,5 & 4,33 & 4,06 & ,944 &, 348 \\
\hline $\mathrm{RU}(\mathrm{X})$ & & & & & & & 3,75 & 3,76 &,- 116 & ,908 \\
\hline
\end{tabular}

Nota: Las diferencias significativas por género han sido marcadas en negrita.

El análisis inferencial solo muestra diferencias significativas por género para el primer ítem $(\mathrm{t}=2,27$; $\mathrm{p}$-Valor=,026), mostrando las chicas un menor grado de satisfacción en cuanto a la adaptación del temario a la nueva situación (PD1). Este mayor número de dudas entre el alumnado femenino queda contrastado en la media obtenida entre ambos géneros al hacer la media de los ítems que componen el bloque. Tras revertir las puntuaciones del ítem PD4, las alumnas obtuvieron una media por debajo de los cuatro puntos $(3,96)$, mientras que los alumnos obtuvieron una media superior a los 4,3, aunque el p-value indica que no hay diferencias significativas entre ambos géneros en la media de los ítems de este primer bloque. Cabe señalar el escaso número de estudiantes que han marcado la opción $\mathrm{NS} / \mathrm{NC}$ en las preguntas de este bloque pues solamente el 1,4 \% lo marcó en la PD2 y el 5,6 $\%$ en la PD4, lo que demuestra que los estudiantes tenían clara su postura respecto a cómo se había gestionado la planificación y desarrollo de la docencia.

Con respecto a los recursos complementarios puestos a disposición del alumnado, son considerados de gran utilidad para una mejor comprensión de los contenidos teóricos por el 81,7 \% de los estudiantes (RU3), al igual que los vídeos incluidos en las presentaciones (RU7) (81,7\%). Destaca el hecho de que prácticamente el mismo porcentaje de estudiantes señala que han tenido mayor dificultad para entender los conceptos mediante la docencia online a través de Microsoft Teams que los que indican que no les ha supuesto mayor dificultad (RU1), pese a que la mayoría indica (55 \%) no haber tenido ningún tipo de problemas de forma general con la aplicación (RU2). Resalta el hecho que prácticamente un $10 \%$ de los estudiantes NS/NC respecto a los problemas con la 
aplicación (RU2), aspecto que llama la atención ya que la respuesta a este item puede considerarse objetiva (no depende de su opinión: o han tenido problemas o no los han tenido). En cuanto al proceso de comunicación alumno-profesor, el $97 \%$ indican que ha sido fluida (R4). Resalta el hecho que ningún estudiante ha marcado las opciones NS/ $\mathrm{NC}$, muy en desacuerdo y en desacuerdo en el ítem R4, lo que afirma el esfuerzo realizado por el profesorado por mantener la comunicación con los estudiantes a pesar de la difícil situación, aunque algo más del 50 \% de los estudiantes considera que la docencia no presencial dificulta su participación (RU5); aun cuando consideran (56\%) que el funcionamiento de los foros y la plataforma Moodle ha sido correcta (RU6).

Finalmente, el test t-student no muestra diferencias significativas por género en ninguna de las cuestiones incluidas en el bloque de recursos. Esta afirmación se corrobora con las puntuaciones medias prácticamente iguales $(3,75$ y 3,76$)$ por género del conjunto de preguntas de este segundo bloque, tras revertir las puntuaciones del ítem RU2.

Con todo ello, la valoración global que el alumnado otorga a esta planificación y capacidad por alcanzar los objetivos pretendidos (VG1) es de 8,1 tanto entre los discentes masculinos como femeninos. Por ello, y con independencia del género $(t=-, 009$ p-Valor=,993) el alumnado evalúa con una calificación de notable alto la subsanación de esta situación de forma rápida y efectiva. Por lo tanto, si bien es cierto que esta situación supone una sobrecarga al profesorado (Silas Casillas y Vázquez Rodríguez, 2020); también es cierto que el alumnado considera cumplido el objetivo marcado de una nueva docencia online que permita un aprendizaje efectivo de acuerdo con García-Planas y Taberna Torres (2020).

\subsection{Visión del alumnado sobre las actividades realizadas y proceso evaluativo establecido}

Respecto a las actividades previstas (preguntas ACT1 a 6) y el sistema de evaluación establecidos (preguntas de la EV1 a la 4), mostrados en la Tabla 7, se confirma esta positiva tendencia entre las respuestas del alumnado hacia la nueva propuesta educativa establecida. 
Tabla 7.

Análisis de las respuestas obtenidas en cada una de las preguntas de los bloques 3: Actividades (ACT) y bloque 4: Evaluación (EV). No sabe/No contesta (NS/NC), muy en desacuerdo (MD), en desacuerdo (D), indiferente (I) de acuerdo (A), muy de acuerdo (MA).

\begin{tabular}{lllllllllll}
\hline Pregunta & $\begin{array}{l}\text { NS/NC } \\
(\%)\end{array}$ & $\begin{array}{l}\text { MD } \\
(\%)\end{array}$ & D (\%) & I (\%) & A (\%) & $\begin{array}{l}\text { MA } \\
(\%)\end{array}$ & Género (X) & T-test & Sig. \\
& & & & & & & 0 & + & Valor & P \\
\hline ACT1 & 2,8 & 1,4 & 2,8 & 11,3 & 45,1 & 36,6 & 4,29 & 3,94 & 1,226 &, 224 \\
ACT2 & 5,6 & 4,2 & 1,4 & 5,6 & 42,3 & 40,8 & 4,24 & 3,86 & 1,077 &, 285 \\
ACT3 & 7,0 & 16,9 & 18,3 & 16,9 & 22,5 & 15,5 & 2,48 & 2,94 & $-1,141$ &, 258 \\
ACT4 & & & 5,6 & 11,3 & 36,6 & 46,5 & 4,33 & 4,2 &, 587 &, 559 \\
ACT5 & & & 2,8 & 12,7 & 40,8 & 43,7 & 4,48 & 4,16 & 1,559 &, 123 \\
ACT6 & 9,9 & 4,2 & 4,2 & 15,5 & 35,2 & 31 & 4 & 3,36 & 1,599 &, 114 \\
ACT(X) & & & & & & & $\mathbf{4 , 1}$ & $\mathbf{3 , 6 7}$ & $\mathbf{2 , 7 0 4}$ &, $\mathbf{0 0 9}$ \\
\hline EV1 & 1,4 & 5,6 & 1,4 & 14,1 & 42,3 & 35,2 & 4,19 & 3,86 & 1,117 &, 268 \\
EV2 & & & & 4,2 & 22,5 & 73,2 & 4,52 & 4,76 & $-1,45$ &, 158 \\
EV3 & 7 & 31 & 21,1 & 14,1 & 16,9 & 9,9 & 2 & 2,46 & $-1,19$ &, 238 \\
EV4 & 1,4 & 18,3 & 18,3 & 16,9 & 19,7 & 25,4 & $\mathbf{2 , 5 7}$ & $\mathbf{3 , 3 4}$ & $\mathbf{- 2 , 1 1}$ &, $\mathbf{0 4}$ \\
EV(X) & & & & & & & 3,89 & 3,59 & 1,442 &, 154 \\
\hline
\end{tabular}

Con respecto a las actividades previstas, más del $81 \%$ del alumnado consideran que han sido adecuadas y suficientes (ACT1) y que han sido organizadas en periodos de tiempo correcto (ACT5) (superior al $84 \%$ ). Además, más del $83 \%$ consideran positivo o muy positivo la utilización de plataformas digitales como Kahoot! para la autoevaluación progresiva de los contenidos aprendidos (ACT2). Sin embargo, no existe una visión clara sobre el aumento en la dificultad de la comprensión de los problemas cuando estos son expuestos de forma online en comparativa con su ejecución en las clases presenciales (ACT3), pero sí que el 83 \% consideran adecuado o muy de acuerdo que la adaptación de las presentaciones preparadas ha permitido la comprensión de los conceptos (ACT4).

Asimismo, el $66 \%$ de los estudiantes está de acuerdo o muy de acuerdo con que los espacios habilitados para el debate online han ayudado a resolver dudas (ACT6), aunque cerca de un $10 \%$ de los estudiantes no tiene una postura clara respecto a esta cuestión (marcan NS/NC) y aunque no existen diferencias significativas entre ambos géneros, parece existir mayores dudas al respecto entre las alumnas (media obtenida 3,36) que entre los alumnos (4). Las diferencias en este ítem, unidas a visiones enfrentadas en ítems como el $\mathrm{ACT}_{3}$, propicia que exista diferencia significativa en la media obtenida entre chicos $(4,1)$ y las chicas $(3,67)$ en el conjunto global de este bloque de preguntas; siendo ésta contundente $(\mathrm{t}=2,704 ; \mathrm{p}$-Valor $=, 009)$.

$\mathrm{El}$ análisis en profundidad de los ítems referidos al sistema evaluativo adoptado, muestra, en líneas generales, un alto grado de satisfacción por los estudiantes coincidente con la tendencia de lo expuesto hasta el momento. Más del $77 \%$ del estudiantado considera el nuevo sistema de evaluación con un mayor porcentaje de seguimiento continuo (alcanzando este el 50 \% de los criterios de evaluación) adecuado (EV1) y más del $95 \%$ considera que la explicación realizada previamente sobre cómo iban a ser 
modificados estos criterios (EV2) ha sido claramente comunicada y explicada por el profesorado. Resalta el hecho que ningún estudiante ha marcado las opciones NS/NC, muy en desacuerdo ni en desacuerdo, lo que corrobora que los criterios de evaluación quedaron claramente expuestos.

Respecto a la prueba final de evaluación, la mayoría del alumnado (un 52 \%) no considera, en líneas generales, que haya sido difícil su realización de manera online (EV3), encontrándose solo un porcentaje inferior al $27 \%$ que sí lo considera; un 14,1\% se muestra indiferente y un $7 \%$ NS/NC. Sin embargo, sí que se encuentra mayor disparidad ante el estrés adicional que esta situación genera (EV4). Para el $45 \%$ del alumnado la evaluación online ha resultado estresante, no resultándolo para el 36 \% y un 16,9 \% se muestra indiferente. Es precisamente en este ítem en el único del bloque en el que aparecen diferencias significativas entre géneros $(t=-2,11 ; p$-Valor $=, 04)$, mostrando las alumnas un nivel mayor de estrés (2,54 en la media obtenida frente al 3,34 de los alumnos, hay que tener presente que este ítem está formulado en negativo). Con todo ello, en este bloque las puntuaciones medias descienden a valores por debajo de los cuatro puntos tanto entre el alumnado masculino $(3,89)$ como el femenino $(3,59)$, pero no existen diferencias significativas entre ellos.

Probablemente, esta mayor repulsa hacia la ejecución de las actividades en línea, y el mayor nivel de estrés ante la realización de los exámenes online esté relacionada con la menor afinidad del alumnado femenino por el uso de las nuevas tecnologías durante el proceso de enseñanza aprendizaje, como ha sido demostrado en estudios comparativos transnacionales como el proyecto ROSE (The Relevance of Science Education) (Sjøberg y Schreiner, 2008).

Esta línea de pensamiento queda confirmada con las respuestas obtenidas en la pregunta global relacionada con este apartado $\left(V_{3}\right)$. La puntuación media en el grado de acuerdo con esta afirmación entre los alumnos es de 7,14. Sin embargo, en el caso de las alumnas, esta media deciente hasta el 5,78. Pese a ello, la diferencia no es significativa $(\mathrm{t}=1,838$; $\mathrm{p}$-Valor $=, 68)$.

\subsection{Percepción del alumnado de la actitud del profesorado y afinidad con el Proyecto}

La Tabla 8 muestra qué visión tiene el alumnado acerca de la actitud del profesorado (preguntas AP $1 \mathrm{a}$ 4) y cómo esta situación les ha afectado emocionalmente (preguntas VE1 a 4). 
Tabla 8.

Resultados porcentuales y análisis inferencial de las preguntas referidas al bloque 5: Actitud del Profesorado (AP) y bloque 6: cómo me he sentido durante este proceso (valoración de los estudiantes (VE). No sabe/No contesta (NS/NC), muy en desacuerdo (MD), en desacuerdo (D), indiferente (I) de acuerdo (A), muy de acuerdo (MA).

\begin{tabular}{lllllllllll}
\hline Pregunta & $\begin{array}{l}\text { NS/NC } \\
(\%)\end{array}$ & $\begin{array}{l}\text { MD } \\
\text { (\%) }\end{array}$ & D (\%) & I (\%) & A (\%) & $\begin{array}{l}\text { MA } \\
(\%)\end{array}$ & Género (X) & T-test & Sig. \\
& & & & & & 0 & + & Valor & P \\
\hline AP1 & & & & 1,4 & 22,5 & 76,1 & 4,62 & 4,8 & $-1,285$ &, 209 \\
AP2 & 1,4 & & 1,4 & 2,8 & 14,1 & 80,3 & 4,48 & 4,78 & $-1,077$ &, 293 \\
AP3 & & & & 1,4 & 19,7 & 78,9 & 4,67 & 4,82 & $-1,116$ &, 274 \\
AP4 & & & & 1,4 & 16,9 & 81,7 & 4,81 & 4,8 &, 084 &, 934 \\
ACT(X) & & & & & & & 4,68 & 4,8 & $-1,117$ &, 274 \\
VE1 & 1,4 & 9,9 & 12,7 & 21,1 & 25,4 & 29,6 & $\mathbf{2 , 7 1}$ & $\mathbf{3 , 8}$ & $\mathbf{- 3 , 2 4 4}$ &, $\mathbf{0 0 2}$ \\
VE2 & 9,9 & 22,5 & 18,3 & 31,0 & 9,9 & 8,5 & 2,38 & 2,32 &, 165 &, 87 \\
VE3 & & 2,8 & 4,2 & 15,5 & 35,2 & 42,3 & 4,33 & 4 & $\mathbf{1 , 2 8 5}$ &, 203 \\
VE4 & 2,8 & 32,4 & 12,7 & 15,5 & 15,5 & 19,7 & $\mathbf{3 , 4 3}$ & $\mathbf{2 , 3 7}$ & $\mathbf{2 , 6 3 2}$ &, $\mathbf{0 1 1}$ \\
VE(X) & & & & & & & $\mathbf{3 , 4 6}$ & $\mathbf{2 , 9 1}$ & $\mathbf{3 , 0 8 5}$ & $\mathbf{, 0 0 3}$ \\
\hline
\end{tabular}

El alumnado valora de forma muy positiva (98,6 \%) el esfuerzo extra que los docentes han tenido que hacer (AP1). Esta valoración del esfuerzo realizado por el profesorado está de acuerdo con lo señalado por otros autores (Silas Casillas y Vázquez Rodríguez; 2020). Así mismo, los estudiantes valoran la atención prestada a través de la tutorización online (AP2) (superior al 94 \%), mostrándose indiferentes a esta cuestión solamente el $2,8 \%$ del alumnado y un porcentaje muy inferior (1,4 \%) en desacuerdo. En esta línea, el alumnado ha percibido la preocupación de los docentes por conocer cómo estaba siendo el proceso de aprendizaje (AP3) (cerca del $99 \%$ ) y más del 98 \% de los estudiantes consideran que sus demandas han atendido satisfactoriamente atendidas (AP4). Destaca el hecho de que ningún estudiante ha marcado NS/NC, muy en desacuerdo (MD) o en desacuerdo (D ) en los ítems AP1, AP3 y AP4 y solamente un 1,4 \% se ha mostrado indiferente en los ítems $\mathrm{AP}_{3}$ y AP4. Las puntuaciones medias de chicos y chicas en este bloque reflejan claramente la satisfacción con la actitud del profesorado (chicos 4,68 y chicas 4,8), no habiendo diferencias significativas entre ambos géneros.

Respecto a cómo se han sentido los alumnos (bloque 6) más del $77 \%$ de los estudiantes señalan haber sentido incertidumbre ante cómo estudiar la asignatura (VE1) y un porcentaje inferior al $19 \%$ de alumnos y alumnas consideran que esta situación favorece unos mejores resultados académicos y el $31 \%$ considera que no afectará a su rendimiento (VE2). Además, de forma general, no han tenido problemas logísticos para poder seguir dicha metodología ( $\mathrm{VE}_{3}$ ) ya que solo un porcentaje inferior al $7 \%$ de alumnos indican haber tenido algún tipo de problema para poder seguir la docencia online. La mayor diversidad de opiniones aparece respecto al futuro de la metodología (VE4), ya que algo más del $32 \%$ está muy en desacuerdo con que la docencia online se adaptará en un futuro mejor a sus necesidades y un porcentaje semejante de estudiantes marca las opciones en desacuerdo, indiferente, de acuerdo y muy de acuerdo. Es en este bloque 
donde aparecen más ítems que muestran diferencias significativas entre las respuestas dadas por los chicos y las chicas. Las alumnas muestran haber tenido significativamente mayor incertidumbre ante cómo afrontar la asignatura $(2,71)$ que el alumnado masculino $(3,8)(V E 1)(t=-3,244 ; p-V a l o r=, 002)$. Además, consideran que esta incertidumbre ante la metodología a usar no quedaría subsanada ni se ajustaría a sus necesidades en una situación de mayor previsión $(3,43)$ a diferencia de los estudiantes masculinos $(2,37)$ (VE4), esta disparidad por género conlleva que exista diferencia significativa en este ítem $(t=2,632 ; p-V a l o r=, 011)$. Los mayores niveles de confusión dada la situación, da lugar a diferencias significativas en las medias obtenidas por género entre el alumnado masculino $(3,46)$ y el femenino $(2,91)$ siendo entre estas medias donde la diferencia entre ambos géneros es mayor $(\mathrm{t}=3,085$; $\mathrm{p}$-Valor $=, 003)$.

De nuevo, esta diferencia evidencia la menor afinidad de las chicas por las nuevas tecnologías en el desarrollo de su actividad pedagógica y su menor confianza en las habilidades que tienen para su uso (Toma et al., 2019). A pesar de las ventajas y resultados positivos que su uso pueda acarrear (Alshammari, 2020; Pensalbe-Rodríguez et al., 2020) no se obtendrán los efectos esperados si su empleo no les resulta (a las chicas) motivante y les agrada (Cope y Ward, 2002; Denessen et al., 2015). Los cambios metodológicos son, posiblemente, peor aceptados por el alumnado femenino dado que implica una innovación de la que son poco afines especialmente las estudiantes universitarias (Osborne y Dillon, 2008; Toma et al., 2019).

Con todo ello, y pese a las dudas lógicas por un nuevo planteamiento pedagógico claramente diferente al que los estudiantes estaban acostumbrados, se evidencia la satisfacción con el trabajo realizado (VG2); hasta tal punto que la puntuación media de los alumnos es de 9,19 sobre 10 y el de las alumnas 9,08, sin existir por tanto diferencias significativas por género ante esta premisa $(\mathrm{t}=, 338$; $\mathrm{p}$-Valor=,736).

\section{Conclusiones}

La actual situación atípica de pandemia propició la necesidad de un cambio a contrarreloj de los planteamientos pedagógicos universitarios. El formato de docencia, las actividades previstas para su desarrollo en el aula o la forma de seguir el proceso de aprendizaje del alumnado tuvieron que cambiar drásticamente. Debido a ello, buscar soluciones se convirtió en algo necesario a alcanzar a la mayor brevedad posible teniendo en cuenta los recursos a disposición. Por ello, y tras una breve instrucción formativa del profesorado, la docencia online se convirtió en la única solución posible y debía ponerse en marcha lo antes posible.

Sin embargo, y pese al corto periodo de reacción, el análisis de los datos obtenidos a través de un cuestionario diseñado para conocer la opinión de los estudiantes respecto a la adaptación de la asignatura «El Medio Natural I: física, química y su didáctica» del grado de Maestro en Educación Primaria de la Facultad de Educación de Albacete, y la metodología empleado en su docencia online muestran, en general, un alto grado de satisfacción. El alumnado ha sido testigo del esfuerzo que la universidad en su conjunto, y en especial los partícipes del presente estudio, tuvieron que llevar a cabo para que se cumplieran los objetivos pedagógicos establecidos. En concreto, el alumnado considera que la docencia online no ha supuesto un detrimento en su rendimiento académico. Los recursos puestos a su disposición (presentaciones, recursos complementarios o simuladores), al igual que los espacios habilitados para la comunicación y la evaluación continua del proceso de aprendizaje, han sido beneficiosos y útiles para el estudio de la asignatura. 
No obstante, a pesar de que tanto el alumnado masculino, como femenino se muestran satisfechos dadas las circunstancias, existen ciertas diferencias por género que no son desdeñables. Los mayores niveles de estrés por parte del alumnado femenino tanto a la hora de afrontar la asignatura, como durante la realización de la prueba final, o el no considerar que con mayor previsión esta metodología puede ser adecuada, evidencian la menor afinidad del género femenino en cuestiones de innovación pedagógica a través de las nuevas tecnologías, a pesar de que estas tecnologías son cada vez más constantes en todos los ámbitos de nuestra vida personal y social.

Ala vista del éxito de la adaptación realizada, podemos concluir que, los primeros pasos hacia una transformación digital de nuestra enseñanza universitaria han sido adecuados, aunque queda un largo camino por recorrer, que pasa por un mejor aprovechamiento de los avances tecnológicos combinado con una profunda revisión metodológica del proceso de enseñanza y un compromiso del profesorado implicado.

\section{Referencias}

Álvarez, Q. (2012) La comunicación no verbal en los procesos de enseñanza-aprendizaje: el papel del profesor. Innovación Educativa, (22), 23-37.

Alshammari, M.T. (2020). Design and evaluation of an adaptive framework for virtual learning environments. International Journal of Advanced and Applied Sciences. 7(5), 39-51. https://doi.org/10.21833/ijaas.2020.05.006

Collado Yurrita, M.A. (2020). RESOLUCIÓN de 11 de marzo de 2020, del Rector de la Universidad de Castilla-La Mancha, por la que se adoptan medidas preventivas y recomendaciones de salud pública relativas a la comunidad universitaria como consecuencia de la situación, evolución y perspectivas del coronavirus (COVID-19). Consejo de Dirección de la Universidad de Castilla-La Mancha. https://www. uclm.es/-/media/Files/Ao1-Asistencia-Direccion/A01-200-Gerencia/COVID-19/ Resolucion-11-de-marzo-rector-medidas-preventivas-firmada.ashx?la=es

Cope, C., y Ward, P. (2002). Integrating learning technology into classrooms: The importance of teachers' perceptions. Journal of Educational Technology \& Society, 5(1), 67-74. https://www.jstor.org/stable/10.2307/jeductechsoci.5.1.67

Denessen, E., Vos, N., Hasselman, F.y Louws, M. (2015). The relationship between primary school teacher and student attitudes towards science and technology. Education Research International, 2015, 7 pages. https://doi.org/10.1155/2015/534690

García-Peñalvo, F. J., Corell, A., Abella-García, V., \& Grande, M. (2020). La evaluación online en la educación superior en tiempos de la COVID-19. Education in the Knowledge Society, 21(12). https://doi.org/10.14201/eks.23013

García-Planas, M.I. y Taberna Torres, J. (2020) Transición de la docencia presencial a la no presencial en la UPC durante la pandemia del COVID-19. International Journal of Educational Research and Innovation, 15, 177-187. https: //doi.org/10.4661/ijeri.5015

González, J. (2006). B-Learning utilizando software libre, una alternativa viable en Educación Superior. Revista Complutense de Educación, 17(1), 121-133. https:// revistas.ucm.es/index.php/RCED/article/view/RCEDo606120121A 
Herrera,M.yPérez,H.(2002).Antecedentes conceptuales, tecnológicosypedagógicospara la propuesta de un modelo educativo a distancia. Estudios Pedagógicos (Valdivia), (28), 157-164. https://dx.doi.org/10.4067/So718-07052002000100009

Hodges, C.,Moore, S.,Lockee, B.,Trust, T. y Bond, A. (2020). The difference between emergency remote teaching and online learning. Educause Review. https:// er.educause.edu/articles/2020/3/the-difference-between-emergency-remoteteaching-and-online-learning

Hurt, B. e Ivergard, T. (2005). E-Learning. A Learning Context in Context. International journal of the computer, the internet and management, 1(1), 18-28.

López-Solera, A. (2020). RESOLUCIÓN de 12 de marzo de 2020, de la Vicerrectora de la Universidad de Castilla-La Mancha, sobre las medidas extraordinarias previstas en coordinación con los vicerrectorados competentes para adaptar la universidad a la suspensión temporal de la actividad educativa presencial. Vicerrectorado de Docencia de la Universidad de Castilla-La Mancha. https://www. uclm.es/-/media/Files/Co1-Centros/CUfcomunicacion/novedades/ResolucionVic-Docencia-medidas-COVID19.ashx

Ministerio de Universidades (2020). Reflexiones sobre criterios generales para la adaptación del sistema universitario español ante la pandemia del Covid-19, durante el curso 2019-2020. https://www.uclm.es/-/media/Files/A01-AsistenciaDireccion/A01-200-Gerencia/COVID-19/Adaptacin-del-sistema-universitarioespaol-a-la-crisis-sanitaria-del-Covid-19--vd-.ashx?la=es

Navarro, C. y Casero, A. (2012). Análisis de las diferencias de género en la elección de estudios universitarios. Estudios sobre educación, 22, 115-132. https://hdl.handle. net/10171/22628

Osborne, J. y Dillon, J. (2008). Science Education in Europe: Critical Reflections. The Nuffield Foundation

Pensalbe-Rodriguez, A., Lopez-Dominguez, E., Hernandez-Velazquez, Y., DominguezIsidro, S. y de la Calleja, J. (2020). Context-Aware Mobile Learning System: Usability Assessment Based on a Field Study. Telematics and Informatics, 48. https://doi.org/10.1016/j.tele.2020.101346

Peñalvo, F. J. (2005). Estado actual de los sistemas e-learning. Teoría de la Educación. Educación y Cultura en la Sociedad de la Información, 6(2). https://doi. org/10.14201/eks.18184

Pruneda, R.E., Castillo, M.C., Mozos, C.M., Muñoz, E.M., Sanz, A.M., Alcázar, A. y Arroyo, L.F. (2019). Moodle: gestión de contenidos online. Ediciones de la Universidad de Castilla-La Mancha. http://doi.org/10.18239/atena.17.2019

Real Decreto 126/2014 de 28 de febrero, por el que se establece el currículo básico de la Educación Primaria. Boletín Oficial del Estado (BOE) 52, Sec I, pág. 19349. https:// www.boe.es/eli/es/rd/2014/o2/28/126/con 
Real Decreto 463/2020, de 14 de marzo, por el que se declara el estado de alarma para la gestión de la situación de crisis sanitaria ocasionada por el COVID-19. Boletín Oficial del Estado (BOE) 67, Sec I, pág 25390. https://www.boe.es/eli/es/ $\mathrm{rd} / 2020 / 03 / 14 / 463 / \mathrm{con}$

Sánchez Santamaría, J., Sánchez Antolín, P. y Ramos Pardo, F.J. (2012). Uso pedagógico de Moodle en la docencia universitaria desde la perspectiva de los estudiantes, Revista Iberoamericana de educación, 60, 15-38. https://doi.org/10.35362/ rie600441

Sánchez, M.I. (2018). Haciauna Educación Primaria de Calidad basada en elm-learning. (Tesis de Maestría). Universidad Oberta de Cataluña (en línea). Murcia

Silas Casillas, J.C y Vázquez Rodríguez, S. (2020). El docente universitario frente a las tensiones que le plantea la pandemia. RLEE Nueva Época (México) Volumen L número especial, 89-120. https://rlee.ibero.mx/plugins/generic/pdfJsViewer/ pdf.js/web/viewer.html?file=https\%3A\%2F\%2Frlee.ibero.mx\%2Findex. php\%2Frlee\%2Farticle\%2Fdownload\%2F97\%2F374\%2F

Sjøberg, S. y Schreiner, C. (2008). Young people, science and technology: Attitudes, values, interests and possible recruitment. ERT: Bruselas.

Toma, R., Ortiz-Revilla, J. y Greca, I. M. (2019). ¿Qué actitudes hacia la ciencia posee el alumnado de Educación Primaria que participa en actividades científicas extracurriculares? Ápice. Revista de Educación Científica, 3(1), 55-69. https://doi. org/10.17979/arec.2019.3.1.4599

Torrecillas, C. (2020). El reto de la docencia online para las universidades públicas españolas ante la pandemia del COVID-19. ICEI Papers COVID-19, (16). https:// eprints.ucm.es/60050/

UCLM (2020). Directrices académicas de adaptación de la evaluación presencial a la no presencial. Comisión de Innovación y Calidad Educativa de la UCLM. https:// www.uclm.es/-/media/Files/Ao1-Asistencia-Direccion/A01-200-Gerencia/ COVID-19/Criterios-academicos-de-adaptacion-de-la-evaluacion-presencial-ano-presencial_UCLM.ashx?la=es

UNESCO. (2020). COVID-19: 10 Recommendations to plan distance learning solutions. Paris: UNESCO. https://bit.ly/34BE6dg 